\title{
Maximum Likelihood Fit of a Gradual Admixture Model to Clines of Gene Frequencies in the Main Islands of Japan
}

\author{
KENICHI AOKI \\ Department of Anthropology, Graduate School of Science, \\ University of Tokyo, Hongo, Bunkyo-ku, \\ Tokyo 113, Japan
}

Received September 7, 1993

\begin{abstract}
The hypothesis was tested that the observed clines of gene frequencies in the main islands of Japan (Kyushu, Shikoku, and Honshu) were produced by a two-phase episode of immigration and admixture. In the first phase, an intrusive population occupies the western part of the main islands, causing a temporary discontinuity in the geographical distribution of the gene frequency at each locus. In the second phase, gradual admixture occurs by the random migration of both immigrants and indigenes, leading to the formation of a cline. Under the assumption that the population density is uniform in space at all times, the process is modelled mathematically as a simple diffusion on a finite linear habitat with reflecting boundaries. The discontinuous gene frequency distribution is approximated by a step function. Maximum likelihood estimates of the parameters of the model are obtained by Marquardt's method, and the chisquare statistic is used to test goodness-of-fit. The model does not give a satisfactory fit at the ABO blood group locus. It does give an acceptable fit at the group-specific component and haptoglobin loci, whether the model fitting is done at each locus separately, or at both loci simultaneously. Moreover, the maximum likelihood estimates of the elapsed time since beginning of admixture are compatible with the proposal that the immigrants arrived at the end of the Jomon period. On the other hand, the maximum likelihood estimates of the eastern limit of initial occupation appear to be too large.
\end{abstract}

Key Words: gene frequency cline, immigration and admixture, maximum likelihood, goodness-of-fit

\section{INTRODUCTION}

Many anthropologists concerned with the origin(s) of modern Japanese regard as important the immigration of rice farmers from the Asian mainland that began roughly 2300 years ago. This theory has recently received strong support from diachronic studies of nonmetric cranial traits (Dodo and Ishida, 1992) and dental characters (Matsumura, 1994), which apparently underwent an abrupt change between the Jomon and Yayoi periods, followed by relative stability afterwards. Consult Hanihara (1991) for a concise account of various contrasting hypotheses. 
Genetic studies of modern Japanese in the main islands of Japan (Kyushu, Shikoku, and Honshu) also provide evidence for the arrival of a population that differed from the indigenes in gene frequencies at several loci. Statistically significant clines, from western Kyushu to northern Honshu, are known for the ABO blood group locus (Nei and Imaizumi, 1966), group-specific component locus (Yuasa et al., 1983), and haptoglobin locus (Omoto, 1986).

Such clines could have been formed, if, as appears to have been the case, an incoming group first settled in the western part of the main islands (e.g., Kanenokuma and Doigahama sites) and subsequently spread eastward. This argument also assumes, often implicitly, that the previous inhabitants were genetically homogeneous. Regional variation in skeletal characters was small during the Jomon period (Yamaguchi, 1982), and this may well have been true of the above genetic systems.

However, it should be noted that numerous processes can lead to the formation of a cline. Hence, the existence of a cline does not immediately imply an episode of immigration and admixture. One way to assess the validity of such a scenario is to define a population genetic model and to test the goodness-of-fit of that model to the observed distribution(s) of gene frequencies. Except for a preliminary attempt by Aoki and Omoto (1980), this has not been done.

In this paper, I present a population genetic model, similar to that of Aoki and Omoto (1980), for the gradual admixture by random migration of two populations that initially differ in the frequency of an allele at one genetic locus and occupy two contiguous regions. The theoretically expected cline derived from the model is a function of several parameters, including the elapsed time since beginning of admixture. At each of the above-mentioned loci, maximum likelihood estimates with errors (standard deviations) are obtained for some of these parameters by fitting the expected to the observed clines. (The other parameters are givens.) The chisquare statistic is used to test the goodness-of-fit of the model to each data set.

Of major interest is the possibility that the observed clines at two or more loci are the result of the same prehistorical event. This hypothesis can be tested by a simultaneous fitting of the model to the data sets at more than one locus. However, in this multi-locus case, the true values of some parameters, e.g., the elapsed time since beginning of admixture, must be the same at all relevant loci. Hence, model fitting is done subject to these constraints.

Before proceeding, I note that the model may appear to be unrealistic in at least two respects. First, it assume that immigration was restricted in time, whereas it may have been spread out over a period of roughly 1000 years (Hanihara, 1987). However, this point is a matter of controversy (e.g., Yamaguchi, 1982). Second, it ignores the higher carrying capacity of farmers relative to hunter-gatherers, which might have generated a wave of advance (Ammerman and Cavalli-Sforza, 1984). However, we are now developing a more realistic model (Aoki et al., submitted), 
which shows that the immigrant farmers are likely to have extended their range by random migration alone (see below for further discussion).

\section{THE MODEL}

I describe a minimal model incorporating the processes that might have produced the observed clines. The episode of immigration and admixture is assumed to comprise two phases. In the first phase, which is relatively short, an intrusive group of farmers occupies the western part of the three main islands of Japan (Kyushu, Shikoku, and Honshu) (see Aoki and Omoto, 1980). In the second phase, which continues up to the present, random migration of both immigrants and indigenes leads to their gradual admixture.

Let us represent the main islands by a continuous linear habitat of length $L$. We use a simple one-dimensional rather than a more realistic two-dimensional model, since our interest is in clines along the long axis of the main islands. The boundaries of the habitat are at Nagasaki in western Kyushu and Aomori in northern Honshu. Distance, indicated by $X$, is measured from Nagasaki as the origin. Hence, Aomori is at distance $L$ from Nagasaki.

Population density is assumed to be uniform in space at all times. It is also assumed to be sufficiently high that the effect of random genetic drift is negligible. Again, this assumption may appear unrealistic, but our theoretical study incorporating logistic growth and conversion of hunter-gatherers to farming (Aoki et al., submitted) shows that admixture of immigrants and indigenes is likely to occur in a region of uniform density.

Consider a selectively neutral allele, and denote its frequency at distance $X$ and time $T$ by $u(X, T)$ (gene frequency distribution). We assume that the gene frequency distribution is initially (i.e., at $T=0$ ) given by the step function,

$$
u(X, 0)=\left\{\begin{array}{l}
v_{1} \text { for } G<X \leqq L \\
v_{2} \text { for } 0 \leqq X \leqq G
\end{array}\right.
$$

The significance of the three parameters, $G, v_{1}$, and $v_{2}$, is as follows. The eastern limit of initial occupation occurs at distance $G$ from Nagasaki; the gene frequency among the native hunter-gatherers is uniformly $v_{1}$; the average gene frequency in the mixed population of immigrants and indigenes weighted by their relative numbers is $v_{2}$.

In the second phase, the spread of immigrant farmers occurs by migration that is random in direction, where the mean and variance of the migration distance per unit time are 0 and $2 D$. ( $D$ is called the diffusion coefficient.) Together with the previous assumptions on population density, this implies that the gene frequency 
distribution, $u(X, T)$, changes according to the diffusion equation,

$$
u_{T}=D u_{X X}
$$

for $0<X<L$ and $T>0$. The subscripts indicate partial differentiation.

To complete the formulation of the model, we posit that there is no migration across the boundaries of the habitat (i.e., on reaching a boundary, direction of motion is reversed) during the second phase. Hence,

$$
u_{X}(0, T)=u_{X}(L, T)=0
$$

The well-known solution of the diffusion equation (2) subject to the boundary conditions (3) and the initial condition (1) is

$$
u(X, T)=v_{1}+\left(v_{2}-v_{1}\right)[(G / L)+2 f(X, T)]
$$

where

$$
f(X, T)=\sum_{n=1}^{\infty}[1 /(n \pi)] \exp \left[-(n \pi)^{2} D T / L^{2}\right] \cos (n \pi X / L) \sin (n \pi G / L) .
$$

\section{MATERIALS AND METHODS}

Allele frequencies at the ABO locus are summarized in Watanabe et al. (1975). Forty-three samples were selected from sites between Nagasaki and Aomori. In each of these samples, deviation from Hardy-Weinberg proportions is nonsignificant. The observed clines for all three alleles are significant at the $1 \%$ level (Nei and Imaizumi, 1966), but only the cline in allele A was examined in this paper. Allele frequencies at the group-specific component and haptoglobin loci were taken from Tables 5 and 6 of Omoto (1986), with the exception of the sample from Kagoshima which was excluded. Statistically significant clines have been reported for allele Gc*2 at the $0.1 \%$ level (Yuasa et al., 1983; Omoto, 1986), and allele $\mathrm{Hp}^{*} 1$ at the 5\% level (Omoto, 1986). These clines were also examined in this paper.

Distance was measured in kilometers along railway routes from Aomori (Nei and Imaizumi, 1966; Omoto, 1986), and the origin was transformed to Nagasaki. The distance between Nagasaki and Aomori measured in this way is $2069 \mathrm{~km}$. Hence, $L=2069$ in our model.

Recall that in Eqs. (4a) and (4b) the expected gene frequency, $u(X, T)$, is given as a function of the variables $X$ and $T$. In the following statistical analysis, it is more appropriate to regard $T$ as a parameter. In fact, there are six parameters, $T, D, L$, $G, v_{1}$, and $v_{2}$, five of which are unknown, occurring in the four nondimensional 
combinations, $t=D T / L^{2}, g=G / L, v_{1}$, and $\Delta v=v_{2}-v_{1}$. Note that $T$ and $D$ cannot be estimated independently since they occur as a product. Fortunately, external estimates of $D$ obtained from ethnographic studies (Ammerman and Cavalli-Sforza, 1984, pp. 78-80) are available.

The maximum likelihood method used for fitting the model to the data set at one locus is as follows. Let us assume that $m$ samples are available, and that the $i$ th sample $(1 \leqq i \leqq m)$ has been obtained at distance $X_{i}$ from Nagasaki. We write the expected gene frequency at distance $X_{i}$ as $u_{i}\left(t, g, v_{1}, \Delta v\right)$, or simply $u_{i}$, and the observed gene frequency there as $p_{i}$. Under the null hypothesis, the distribution of $p_{i}$ is binomial with mean $u_{i}$ and variance $u_{i}\left(1-u_{i}\right) / N_{i}$, where $\mathrm{N}_{i}$ is the number of genes sampled at $X_{i}$. By the arcsin transformation,

$$
\begin{aligned}
& \bar{z}_{i}=2 \arcsin \left(u_{i}^{\frac{1}{2}}\right), \\
& z_{i}=2 \arcsin \left(p_{i}^{\frac{1}{2}}\right),
\end{aligned}
$$

this distribution is rendered approximately normal, and the variance becomes a known constant, $1 / N_{i}$, that is independent of the parameters (Sokal and Rohlf, 1981, p. 427).

Hence, the likelihood that the observed gene frequency is $p_{i}$ at $X_{i}(1 \leqq i \leqq m)$ becomes

$$
Q=\prod_{i=1}^{m}\left[N_{i} /(2 \pi)\right]^{\frac{1}{2}} \exp \left[-N_{i}\left(z_{i}-\bar{z}_{i}\right)^{2} / 2\right] .
$$

(Likelihood is denoted by $Q$ rather than the usual $L$, since we have already used $L$ for the length of the habitat.) Maximizing Eq. (6) is equivalent to minimizing

$$
S=\sum_{i=1}^{m} N_{i}\left(z_{i}-\bar{z}_{i}\right)^{2} .
$$

Maximum likelihood estimates of the four composite parameters, $t, g, v_{1}$, and $\Delta v$ were obtained by Marquardt's method (Press et al., 1992, p. 678). In implementing this method, it was necessary to avoid negative values of $t$, since otherwise the series (4b) diverges. Marquardt's method was supplemented by grid search to ensure that the global rather than a local minimum of Eq. (7) had been reached.

When fitting the model to the data sets at more than one locus, the expected gene frequencies were computed at each locus, with the parameters $v_{1}$ and $\Delta v$ free to vary among the loci, but the parameters $t$ and $g$ constrained to take the same values. The sum in Eq. (6) or (7) is over all samples at all loci. I only had occasion to apply this method to the data sets at two loci: group-specific component and haptoglobin. In this case, six parameters were estimated. 
Goodness-of-fit of the model can be tested by noting that $S$ at its minimum has a chisquare distribution (see Eq. (7)). The number of degrees of freedom equals the number of samples minus the number of estimated parameters.

\section{RESULTS}

Summarized below are the results of model fitting to the data sets at each locus separately. Maximum likelihood estimates of the four composite parameters are given with estimates of their errors (standard deviations), the associated chisquare value, the number of degrees of freedom, and the level of significance.

(1) Allele $\mathrm{A}$ at the $\mathrm{ABO}$ blood group locus:

$$
\begin{aligned}
& t=0.0538 \pm 2.703 \\
& g=0.9298 \pm 113.5 \\
& v_{1}=0.1071 \pm 292.6 \\
& \Delta v=0.1808 \pm 292.6 \\
& \chi^{2}=262.44, \text { d.f. }=39, P<0.001
\end{aligned}
$$

The chisquare value is highly significant and the model is rejected. Hence, the maximum likelihood estimates of the parameters are meaningless.

(2) Allele Gc*2 at the group-specific component locus:

$$
\begin{aligned}
& t=0.0225 \pm 5.919 \\
& g=0.0343 \pm 514.7 \\
& v_{1}=0.2493 \pm 0.0066 \\
& \Delta v=0.3121 \pm 4686 \\
& \chi^{2}=2.12, \text { d.f. }=7,0.95<P<0.975
\end{aligned}
$$

The chisquare value is small and the model is acceptable. However, the large errors attached to the maximum likelihood estimates of $t, g$, and $\Delta v$ make these estimates unreliable. This outcome is apparently due to the flatness of the likelihood surface.

(3) Allele Hp*1 at the haptoglobin locus:

$$
\begin{aligned}
& t=0.0035 \pm 0.0096 \\
& g=0.7823 \pm 0.0522 \\
& v_{1}=0.2331 \pm 0.0072
\end{aligned}
$$




$$
\begin{aligned}
& \Delta v=0.0266 \pm 0.0085 \\
& \chi^{2}=23.00, \text { d.f. }=15,0.05<P<0.10
\end{aligned}
$$

The chisquare value is not significant and the model is acceptable. Moreover, the small errors place reliable bounds on the maximum likelihood estimates.

In order to obtain the maximum likelihood estimate of the elapsed time since beginning of admixture, $T$, we require an independent estimate of the diffusion coefficient, D. Ammerman and Cavalli-Sforza (1984, pp. 78-80) review data from various ethnographic studies of hunter-gatherers and farmers. They suggest a range of possible values between 10 and 100 square kilometers per year. Hence, recalling $L=2069$, we conclude that $T$ lies between 149.8 and 1498 years. Our estimate for $G$ is 1619 kilometers, placing the eastern limit of the initial occupation in the vicinity of Fukushima.

Since the observed clines at the group-specific component and haptoglobin loci were found to be consistent with the model, I next attempted to fit the model simultaneously to both data sets (Fig. 1). The result is summarized below.
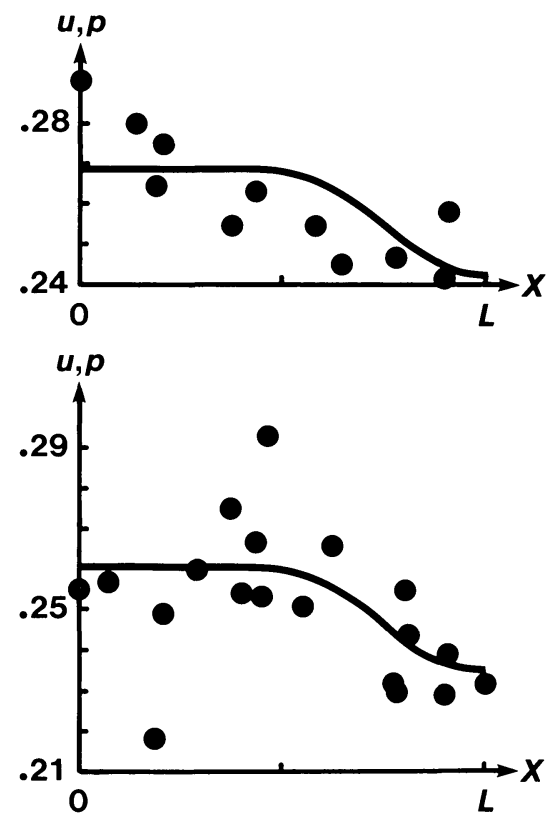

Fig. 1. Simultaneous maximum likelihood fit of the model to the data sets at the group-specific component (upper) and haptoglobin (lower) loci. Horizontal axis is the railway distance from Nagasaki, where $L=2069 \mathrm{~km}$. Vertical axis is the expected $(u)$ or observed $(p)$ gene frequency. Dots indicate the observed allele frequencies of $\mathrm{Gc}^{* 2}$ (upper) and $\mathrm{Hp}^{* 1}$ (lower). Curves indicate the expected gene frequency distribution at each locus. 
(4) Allele Gc*2 at the group-specific component locus and allele $\mathrm{Hp}^{* 1}$ at the haptoglobin locus:

$$
\begin{aligned}
& t=0.0079 \pm 0.0132 \\
& g=0.7358 \pm 0.0898 \\
& v_{1}\left(\mathrm{Gc}^{*} 2\right)=0.2423 \pm 0.0124 \\
& \Delta v(\mathrm{Gc} * 2)=0.0263 \pm 0.0145 \\
& v_{1}\left(\mathrm{Hp}^{*}\right)=0.2344 \pm 0.0095 \\
& \Delta v(\mathrm{Hp} * 1)=0.0263 \pm 0.0107 \\
& \chi^{2}=37.34, \text { d.f. }=24,0.025<P<0.05
\end{aligned}
$$

The chisquare value is significant at the 5\% level. Nevertheless, Press et al. (1992, p. 654) suggest that the model need not be rejected, even at lower probabilities, if the distributions of the observed gene frequencies around the expected values are not strictly normal.

If we choose to accept the model, then our maximum likelihood estimate of $T$ lies in the range between 338.2 and 3382 years depending on the value assumed for $D$. The maximum likelihood estimate of $G$ is 1522 kilometers from Nagasaki, which means that the immigrant farmers reached the northern Kanto area in the first phase of colonization.

\section{DISCUSSION}

The simple model of gradual admixture described here did not give an acceptable fit to the observed cline in allele $\mathrm{A}$ at the $\mathrm{ABO}$ blood group locus. In a previous study, Aoki and Omoto (1980) suggested that this cline might have been formed by an episode of immigration and admixture triggered by the arrival of a foreign group at the end of the Jomon period. However, their claim, lacking a statistical test for goodness-of-fit, was premature. It is also worth noting that the frequency of allele A in Kyushu and western Honshu is higher than in any of the surrounding populations on the Asian mainland. Since the model assumes that the frequency was even higher among the immigrant farmers, there would be no suitable candidate for a source population.

On the other hand, the model did given an acceptable fit to the observed clines in allele $\mathrm{Gc}^{* 2}$ at the group-specific component locus and in allele $\mathrm{Hp}^{* 1}$ at the haptoglobin locus. This was true whether model fitting was done at each locus separately, or at both loci simultaneously. Moreover, the maximum likelihood estimates of the elapsed time since beginning of admixture are not incompatible with the proposed timing. In particular, simultaneous fitting to the data sets at both loci yielded values of $T$ in the range of several hundred to several thousand years. 
However, it should be noted that the errors attached to the maximum likelihood estimates are large, especially at the group-specific component locus.

When the model is consistent with the data set at a locus, we are justified in searching for a modern population on the Asian mainland with a gene frequency that is more extreme than the estimated value for the mixed population of immigrants and indigenes, i.e., $v_{2}$. Presumably, they are descended from the same ancestral population that produced the immigrants to Japan. In this regard, Yuasa et al. (1983), on the basis of information on allele $\mathrm{Gc}^{*} 2$, give a plausible argument locating a possible homeland in north Asia.

Finally, a word on the maximum likelihood estimates of $G$, which appear to be too large. If these estimates are valid, then the initial occupation by the immigrant farmers must have extended as far as the northern Kanto or southern Tohoku regions. On the other hand, the estimates may be artifacts of a model that is too simple. However, our theoretical study (Aoki et al., submitted) suggests the assumptions of the present model closely approximate the conditions obtaining under a more realistic model incorporating logistic growth and conversion of huntergatherers to farming. Hence, this perplexing outcome cannot easily be attributed to this cause and must remain a mystery for now.

\section{ACKNOWLEDGEMENTS}

I thank M. Shida and Y. Mizoguchi for useful suggestions on iterative solutions of maximum likelihood problems.

\section{REFERENCES}

Ammerman, A.J., and Cavalli-Sforza, L.L. (1984) The Neolithic Transition and the Genetics of Populations in Europe, Princeton Univ. Press, Princeton, New Jersey.

Aoki, K., and Omoto, K. (1980) An analysis of the ABO gene frequency cline in Japan: A migration model. J. Anthrop. Soc. Nippon 88, 109-122.

Aoki, K., Shida, M., and Shigesada, N. Travelling wave solutions for the spread of farmers into a region occupied by hunter-gatherers. (submitted)

Dodo, Y., and Ishida, H. (1992) Consistency of nonmetric cranial trait expression during the last 2,000 years in the habitants of the central islands of Japan. J. Anthrop. Soc. Nippon 100, 417-423.

Hanihara, K. (1987) Estimation of the number of early migrants to Japan: A simulative study. $J$. Anthrop. Soc. Nippon 95, 391-403.

Hanihara, K. (1991) Dual structure model for the population history of the Japanese. Japan Review 2, 1-33.

Matsumura, H. (1994) A microevolutional history of the Japanese people from a dental characteristics perspective. Anthropol. Sci. 102, 93-118.

Nei, M., and Imaizumi, Y. (1966) Genetic structure of human populations. I. Local differentiation of blood group gene frequencies in Japan. Heredity 21, 9-35.

Omoto, K. (1986) The distribution of red cell enzyme and serum protein types in a sample from Iwate, northern Japan, with the description of geographical cline in Gc subtypes. J. Anthrop. Soc. Nippon 94, 51-63. 
Press, W.H., Teukolsky, S.A., Vetterling, W.T., and Flannery, B.P. (1992) Numerical Recipes in Fortran, 2nd Ed., Cambridge Univ. Press, Cambridge.

Sokal, R.R., and Rohlf, F.J. (1981) Biometry, 2nd Ed., Freeman, New York.

Watanabe, S., Kondo, S., and Matsunaga, E., ed. (1975) JIBP Synthesis: Human Adaptability, Vol. 2, Anthropological and Genetic Studies on the Japanese, Univ. of Tokyo Press, Tokyo.

Yamaguchi, B. (1982) A review of osteological characteristics of the Jomon population in prehistoric Japan. J. Anthrop. Soc. Nippon 90 (Suppl.), 77-90.

Yuasa, I., Saneshige, Y., and Okada, K. (1983) Geographical cline of allele frequency of group-specific component $(\mathrm{Gc})$ in the Japanese populations: An analysis of data obtained by immunoelectrophoresis. Jpn. J. Human Genet. 28, 255-261. 